\title{
Attosecond Molecular Angular Streaking with All-Ionic Fragments Detection
}

\author{
Wei Quan $\odot,{ }^{1,2}$ Vladislav V. Serov $\odot,{ }^{3}$ MingZheng Wei, ${ }^{1}$ Meng Zhao, ${ }^{1,2}$ Yu Zhou, ${ }^{1,2}$ YanLan Wang, ${ }^{1}$ \\ Xuan Yang Lai, ${ }^{1,2}$ Anatoli S. Kheifets, ${ }^{4, *}$ and XiaoJun Liu ${ }^{1,2, \dagger}$ \\ ${ }^{1}$ State Key Laboratory of Magnetic Resonance and Atomic and Molecular Physics, Wuhan Institute of Physics and Mathematics, \\ Innovation Academy for Precision Measurement Science and Technology, Chinese Academy of Sciences, Wuhan 430071, China \\ ${ }^{2}$ University of Chinese Academy of Sciences, Beijing 100049, China \\ ${ }^{3}$ Department of Theoretical Physics, Saratov State University, 83 Astrakhanskaya, Saratov 410012, Russia \\ ${ }^{4}$ Research School of Physics, The Australian National University, Canberra, Australian Capital Territory 2601, Australia
}

(Received 27 July 2019; published 26 November 2019)

\begin{abstract}
Attosecond angular streaking (or "attoclock") is an insightful technique for probing the ultrafast electron dynamics in strong laser fields. Up until recently, this technique relied solely on an accurate measurement of the photoelectron momentum distribution and has remained restricted to atomic targets. Here, we propose a novel attosecond angular streaking scheme applicable to molecules, for which the ionic fragments of dissociative ionization are detected in the polarization plane of a close-to-circular polarized laser light. Our ionic attoclock measurements are consistent with theoretical results from a numerical solution of the time-dependent Schrödinger equation and an upper bound of 10 as on the tunneling time from the attoclock readings in the $\mathrm{H}_{2}$ molecule has been given, which is significantly smaller than any definitions of tunneling time available in the literatures.
\end{abstract}

DOI: 10.1103/PhysRevLett.123.223204

Time-resolved measurements of ultrafast atomic and molecular dynamics are conventionally performed in pump-probe experiments. For this conventional scheme, two ultrashort laser pulses are used and the ultimate time resolution is determined by the pulse duration. Recent advances in ultrafast laser science have achieved production of ultrashort attosecond pulses [1,2]; however, the available attosecond light pulses so far are still not intense enough to implement fully a pump-probe experiment. As a substitute, the attosecond pump and femtosecond probe measurements have been performed in which the controllable pump-probe delay is converted either to photoelectron energy [3] or yield [4], thus allowing attosecond timeresolved studies of atomic and molecular ionization. At the same time, one has witnessed the emergence and rapid development of a self-referencing technique known as attosecond angular streaking (or attoclock) [5-8]. Even though it is conducted with femtosecond pulses, it is capable of resolving photoelectron dynamics with attosecond precision. This technique is based on measuring an offset angle of the peak photoelectron momentum distribution in the polarization plane of a close-to-circular polarized laser pulse. The attoclock attempts to relate this offset angle with the time the tunneling electron spends under the barrier (tunneling time) [5-8]. As the tunneling ionization is an exponentially suppressed process, it starts most probably at the maximum of the driving laser pulse. The electric field of the laser pulse is aligned with the major axis of the polarization ellipse at this instant. It is expected that the photoelectron emerges from the tunnel with zero velocity (the adiabatic hypothesis) and its kinetic momentum captures the vector potential of the laser field at the time of exit. This momentum is carried to the detector and its angular displacement relative to the minor polarization axis is converted to the tunneling time with an impressive conversion rate of $\sim 7$ as per one degree of rotation at $\lambda \sim 800 \mathrm{~nm}$.

Recently, an alternative interpretation of the attoclock measurements has been proposed and it is believed that the attoclock offset angle is wholly or partially attributed to the photoelectron scattering in the Coulomb potential of the ionic core [9-11] while the tunneling process is instantaneous. This is in stark contrast to those works which advocate a finite tunneling time $[12,13]$ as opposite to zero tunneling time $[9,10,14,15]$. Much of this controversy stems from the present interpretation of the attoclock measurements that relies heavily on the assumptions of the initial conditions of the photoelectron right after the tunneling, such as its momentum and position, which, in turn, depends on the specific laser parameters and atomic targets employed in various experiments.

While the debate of the finite tunneling time is still open, it is thought provoking to extend attoclock measurements to more complex targets such as molecules and to apply the same arguments that support, or eliminate, the tunneling time in atoms. Indeed, some preliminary attoclock measurements on molecule $\mathrm{H}_{2}$ have been conducted using dissociative single [16] or double [17] ionization processes. In both of these works, photoelectrons were detected in coincidence with fragmenting ions, as in the conventional 


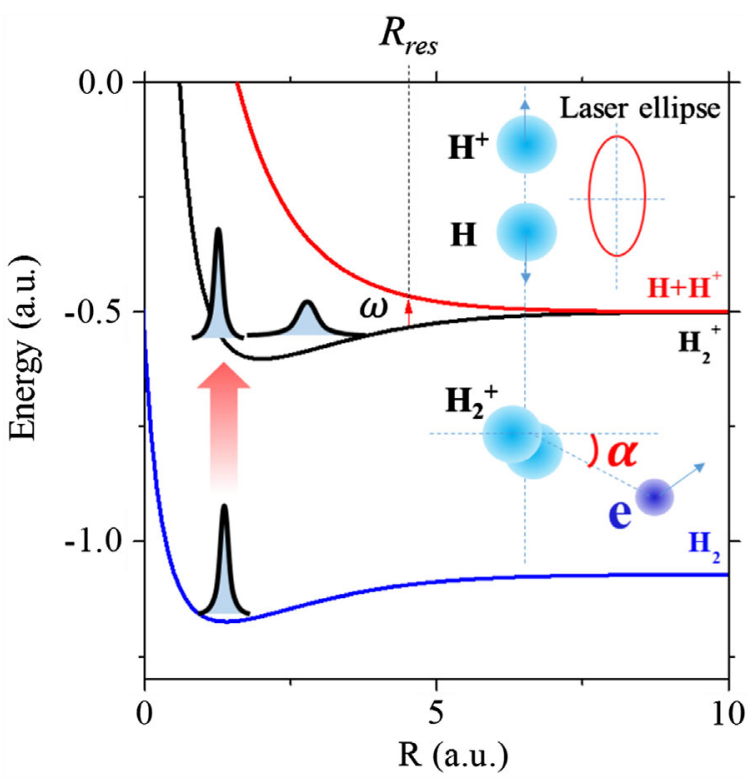

FIG. 1. Graphical representation of the molecular attosecond angular streaking scheme. When subjected to an elliptically polarized laser pulse, the $\mathrm{H}_{2}$ molecule at its equilibrium interatomic separation is tunnel ionized and the $\mathrm{H}_{2}^{+}$ion starts to expand. At the internuclear separation $R_{\text {res }}$, when $E\left(1 s \sigma_{g}\right)-$ $E\left(2 p \sigma_{u}\right)=\omega$, a transition to the $\mathrm{H}_{2}^{+}$dissociative state takes place. The dissociation of $\mathrm{H}_{2}^{+}$is strongly aligned with the electric field vector, i.e., the major polarization axis, providing a natural frame in which the attoclock offset angle of $\mathrm{H}_{2}^{+}$ion can be read.

atomic attoclock scheme. However, no discussions of the finite tunneling time have been made.

In this Letter, we propose and demonstrate on $\mathrm{H}_{2}$ a novel molecular attoclock scheme which is based solely on the ionic fragments detection, the $\mathrm{H}_{2}^{+}$ions, and the protons $\mathrm{H}^{+}$. This scheme has the advantages to eliminate some of the difficulties encountered in the conventional atomic attoclock implementations as discussed above. The proposed ionic attoclock is very robust as it does not require the knowledge of the exact orientation of the polarization ellipse, and more importantly, it does not rely on the adiabatic hypothesis of electron tunneling. This hypothesis has raised the ongoing controversy of the tunneling delay time in the current strong field and attosecond community $[9,10,12-15]$. Resorting to a numerical solution of the timedependent Schrödinger equation (TDSE) and its comparison with the ionic attoclock measurements, we further address the question of a finite tunneling time in $\mathrm{H}_{2}$ and give an upper bound on the tunneling time from the attoclock readings in the $\mathrm{H}_{2}$ molecule.

The principle of this novel attoclock scheme is illustrated in Fig. 1. When subjected to an elliptically polarized fewcycle laser pulse, one bound electron of $\mathrm{H}_{2}$ tunnel ionizes and gives rise to a photoelectron and the $\mathrm{H}_{2}^{+}$ion. Because the composite system $\mathrm{H}_{2}^{+}+e^{-}$is neutral, its center of mass is not affected by the driving laser pulse. One can also argue
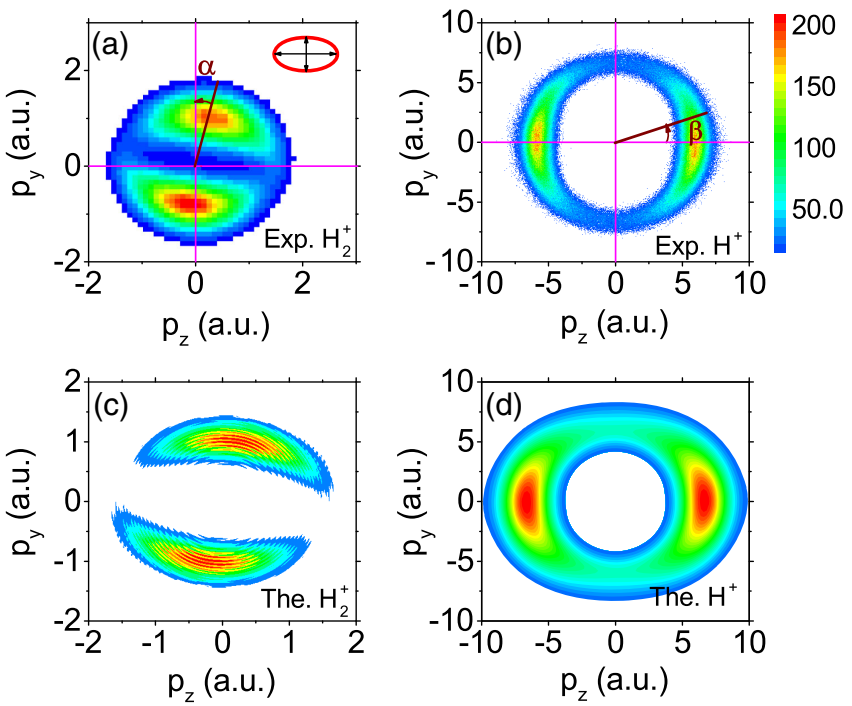

FIG. 2. Experimental (top row) and simulated (bottom row) momentum distributions of the $\mathrm{H}_{2}^{+}$(left) and $\mathrm{H}^{+}$(right) in the polarization plane of an elliptically polarized few-cycle laser pulse with a center wavelength of $760 \mathrm{~nm}$, a peak intensity of $4.6 \times 10^{14} \mathrm{~W} / \mathrm{cm}^{2}$ and an ellipticity of 0.70 . The major axis of the polarization ellipse (electric field $E$ ) is horizontal whereas the minor axis (vector potential $A$ ) is vertical. The angles $\alpha$ and $\beta$ are defined between the emission directions of $\mathrm{H}_{2}^{+}(\alpha)$ and $\mathrm{H}^{+}(\beta)$ relative to the minor and major axes of the polarization ellipse, respectively, as indicated in panels (a) and (b).

that the momentum transferred by the laser field is negligible and the recoil momentum of $\mathrm{H}_{2}^{+}$compensates the departing photoelectron momentum. Hence, the attoclock offset angles of the photoelectron and the $\mathrm{H}_{2}^{+}$ion in the polarization plane are identical. Except for this offset angle, both the electron and the $\mathrm{H}_{2}^{+}$ion are aligned with the vector potential pointing along the minor polarization axis in the correspondingly measured momentum distributions in the polarization plane. At the same time, the dissociation of $\mathrm{H}_{2}^{+}$is strongly aligned with the electric field vector, i.e., the major polarization axis. We confirm that this alignment is rigid and, for short ionizing pulses, it is not affected by intensity dependent offsets. Thus, it provides a natural frame in which the attoclock offset angle can be determined. Compared to the conventional attoclock measurement scheme, which unavoidably depends on the assumption of the initial conditions of photoelectrons, the ionic attoclock is not based on the adiabatic tunneling scenario as the $\mathrm{H}_{2}^{+}$ion is naturally at rest when the photoelectron emerges from under the barrier.

The experiment is performed using a cold target recoilion momentum spectroscopy (COLTRIMS) [18]. Elliptically polarized pulses with a duration of about $5.3 \mathrm{fs}$, a center wavelength of $760 \mathrm{~nm}$, and a repetition rate of $5 \mathrm{kHz}$, which were produced by a Ti:sapphire based laser system and a hollow-fiber compressor, were focused onto $\mathrm{H}_{2}$ molecules inside our COLTRIMS setup [19-21] 


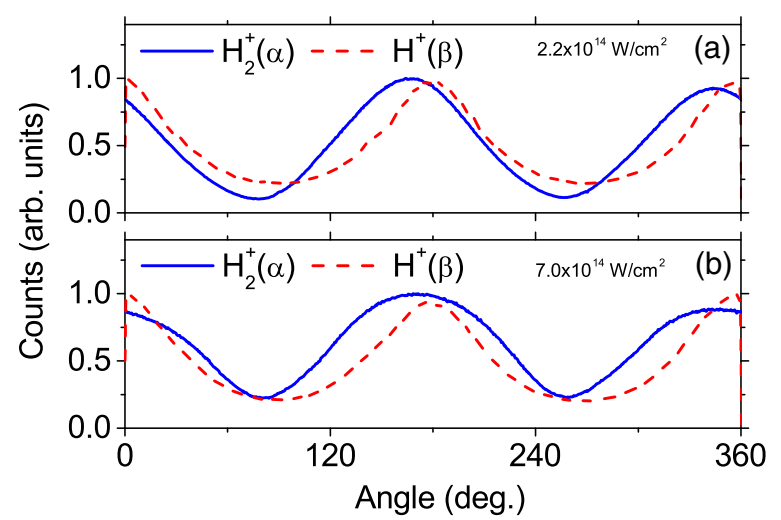

FIG. 3. Radially integrated momentum distributions of $\mathrm{H}_{2}^{+}$ (blue) and $\mathrm{H}^{+}$(red) at the field intensities of $2.2 \times 10^{14} \mathrm{~W} / \mathrm{cm}^{2}$ (top) and $7.0 \times 10^{14} \mathrm{~W} / \mathrm{cm}^{2}$ (bottom).

(see Supplemental Material [22]). The three-dimensional momenta of the produced photofragments (i.e., $\mathrm{H}_{2}^{+}$and $\mathrm{H}^{+}$ ions) can be retrieved from the recorded time-of-flight and impact positions on the detector.

The typical measured and calculated ionic fragment momentum distributions in the polarization plane are illustrated in Fig. 2. The left column shows the $\mathrm{H}_{2}^{+}$ distribution whereas the right column visualizes the same distribution of $\mathrm{H}^{+}$. Experimental and theoretical distributions are exhibited on the top and bottom rows, respectively. Here, we define angles $\alpha$ and $\beta$ which characterize the emission directions of $\mathrm{H}_{2}^{+}(\alpha)$ and $\mathrm{H}^{+}(\beta)$ relative to the minor and major axes of the polarization ellipse, respectively, as indicated in Figs. 2(a) and 2(b). Here, the field intensity is estimated to be $4.6 \times 10^{14} \mathrm{~W} / \mathrm{cm}^{2}$. We see that the $\mathrm{H}_{2}^{+}$distribution is aligned closely with the vector potential $A$ (minor polarization axis, vertical in the figure, which is also shown in the inset of Fig. 2) whereas the $\mathrm{H}^{+}$distribution is aligned with the $E$ field (major axis, horizontal in the figure).

In Fig. 3 we further plot the radially integrated momentum distributions of $\mathrm{H}_{2}^{+}$and $\mathrm{H}^{+}$with respect to $\alpha$ and $\beta$, from which the attoclock offset angles, $\alpha_{\max }$ and $\beta_{\max }$, can be revealed easily. They are identified as the angular displacement of the maxima in the $\mathrm{H}_{2}^{+}$distribution relative to minor axis of the polarization ellipse $\left(\alpha_{\max }\right)$ and the maxima in the $\mathrm{H}^{+}$distribution relative to the major axis $\left(\beta_{\max }\right)$. The resulting offset angles are displayed in Fig. 4 along with our theoretical predictions.

The theoretical modeling for the $\mathrm{H}_{2} \rightarrow \mathrm{H}_{2}^{+}+e$ process is based on a numerical solution of a three-dimensional (3D) TDSE in the single-active electron Hartree-Fock approximation [23]. In this approach, the full complexity of the molecular potential as well as an indirect part of interelectron interaction is taken into account. The only process that is neglected is an excitation of the bound electron; i.e., the $\mathrm{H}_{2}^{+}$ion remains in its ground state (see Fig. 1 for graphical illustration). A similar modeling for

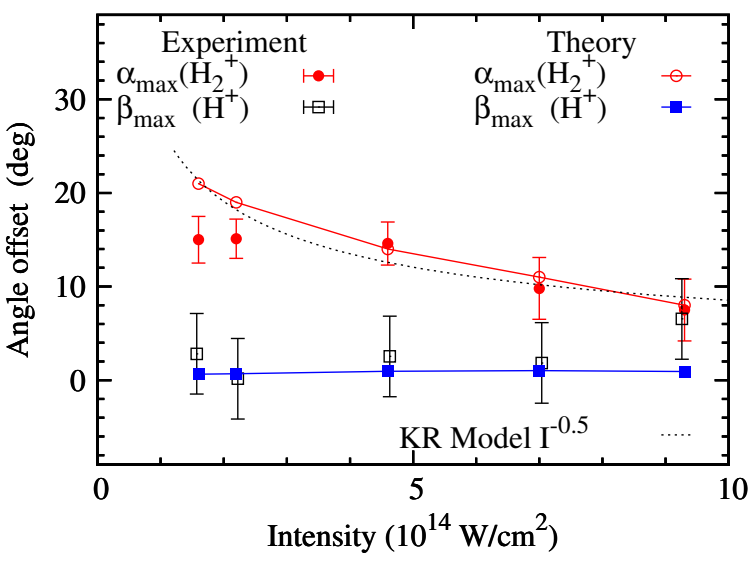

FIG. 4. The molecular attoclock offset angles $\alpha_{\max }$ in the $\mathrm{H}_{2}^{+}$ angular distribution and $\beta_{\max }$ in the $\mathrm{H}^{+}$angular distribution from the TDSE calculations (theory) and the experiment. The dotted line visualizes the prediction of the KR model $\alpha_{\max } \propto I^{-0.5}$.

$\mathrm{H}_{2}^{+}+\hbar \omega \rightarrow \mathrm{H}+\mathrm{H}^{+}$is based on a numerical solution of the 4D TDSE with the full account for the nuclear motion [24]. Detailed description of theoretical simulations is given in the Supplemental Material [22].

Both the theory and experiment, which are rather close, show the same tendency of the decreasing attoclock offset angle $\alpha_{\max }$ with the growing field intensity. This decrease has been already documented in atomic attoclock measurements. It can be interpreted either as a decreasing tunneling time across a narrowing potential barrier $[5,6]$, or a weaker Coulomb scattering of a faster photoelectron on the residual ion [11]. In the latter work, the so-called Keldysh-Rutherford (KR) model is proposed in which the point of the closest approach in the Rutherford scattering formula is equated with the Keldysh tunnel width $b=$ $I_{p} / E_{0}$ expressed via the ionization potential $I_{p}$ and the peak electric field $E_{0}$. At the laser pulse intensities under consideration, $E_{0} \ll 1$ a.u. and hence the tunnel width is significantly larger than the interatomic distance $b \gg R$. As the KR model neglects a possible rescattering of the photoelectron on the parent ion, the molecular orientation is of little effect under the present experimental conditions. The signature of the KR model is a characteristic field intensity dependence of the offset angle $\alpha_{\max } \propto I^{-0.5}$ which is also exhibited in Fig. 4. Both the theoretical and the experimental data, within the error bars, conform with this intensity dependence thus indicating the Coulomb origin of the offset angle and pointing to the instantaneous tunneling.

We further support this scenario by conducting a similar set of TDSE calculations on a Yukawa molecule $Y_{2}$ made of two Yukawa atoms with a screened Coulomb potential $U(r)=-(Z / r) \exp (-r / a)$ with $Z=1$ and $a=2.187$. Both the internuclear distance $R=1.4$ a.u. and the ionization potential $I_{p}=15.6 \mathrm{eV}$ in $Y_{2}$ are identical to those of $\mathrm{H}_{2}$. The largest attoclock offset angle in $Y_{2}$ across the studied intensity range is not exceeding $1.5^{\circ}$. This gives an 
upper bound on the molecular tunneling time of $\sim 10$ as. Even though this is considerably larger than the analogous estimates for the Yukawa atom, $0.25^{\circ}$ and 1.8 as, respectively [10], it attributes nearly whole of the attoclock offset angle in $\mathrm{H}_{2}$ to the photoelectron Coulomb scattering. It also rules out commonly used theoretical definitions of tunneling time which run in the tens to few hundreds of attoseconds [25].

The molecular attoclock angle $\beta_{\max }$ is rather small, not exceeding $2^{\circ}$ in theory and matching that within the experimental error bars. This indicates the validity of the axial recoil approximation which had been questioned in [26]. In the latter work, a noticeable rotation of the $\mathrm{H}_{2}^{+}$ion prior to its dissociation, of the order of $10^{\circ}$, was detected. However, the previous work [26] employed rather long pulses of 25 fs duration, about $5 \times$ longer than in the present study. Respectively, the rotation angles of the $\mathrm{H}_{2}^{+}$ion in [26] were found greater by a similar factor.

In conclusion, we demonstrated the utility of the molecular hydrogen attoclock based on the sole detection of the ionic $\mathrm{H}_{2}^{+}$and $\mathrm{H}^{+}$fragments. Such an attoclock measurement returns essentially the same offset angles as a conventional attoclock based on detection of the peak photoelectron momentum distribution. The advantage of the ionic attoclock is the natural alignment of the $\mathrm{H}^{+}$ momentum distribution in the major axis of the polarization ellipse which may not be known sufficiently well. In addition, the adiabatic hypothesis of the zero velocity of the tunneling electron at the exit is not needed to interpret the reading of the ionic attoclock. The $\mathrm{H}_{2}^{+}$ion is naturally at rest when the photoelectron exits the tunnel. Our experimental results and supporting TDSE calculations along with predictions of a classical Coulomb scattering model point to instantaneous molecular tunneling similarly to the atomic hydrogen case [10]. This is further supported by TDSE simulations on a Yukawa molecule with a screened Coulomb potential which return vanishingly small attoclock offset angles.

In future experiments, the molecular attoclock with allionic fragment detection may be extended to other diatomic molecules beyond the simplest $\mathrm{H}_{2}$ in which tunnel ionization is followed by a fragmentation process. For example, it might be insightful to apply this technique to $\mathrm{N}_{2}$ with a bonding ground state as well as to $\mathrm{O}_{2}$ with an antibonding ground state and to see if the ground state symmetry plays any role in the reading of the ionic attoclock. Another group of targets of particular interest are the diatomic noble gas clusters $\mathrm{Ne}_{2}, \mathrm{Ar}_{2}$ which have a very large interatomic separation. Because of this large separation, the photoelectron momentum distribution in the polarization plane shows a very prominent interference pattern. This pattern can be controlled by selecting the gerade or ungerade symmetry of the ionic dissociative state [27]. It will be intriguing to explore these effects in the ionic attoclock settings and especially, by comparing the angular offsets of the ions, one expects to obtain additional information on the time delay of the two dissociative channels.

This work is supported by the National Key Research and Development Program of China (No. 2019YFA0307702), the National Natural Science Foundation of China (No. 11527807, No. 11774387, No. 11834015, No. 11847243, No. 11804374 , No. 11874392, No. 11974383), the Strategic Priority Research Program of the Chinese Academy of Sciences (No. XDB21010400). A.S. K. acknowledges support of the Chinese Academy of Sciences (CAS) under the CAS President's International Fellowship Initiative (PIFI, No. 2018VMA0021). Both A. S. K. and V. V. S. wish to thank the Wuhan Institute of Physics and Mathematics of CAS for warm hospitality.

\section{*A.Kheifets@anu.edu.au} †jliu@wipm.ac.cn

[1] P. M. Paul, E. S. Toma, P. Breger, G. Mullot, F. Augé, Ph. Balcou, H. G. Muller, and P. Agostini, Observation of a train of attosecond pulses from high harmonic generation, Science 292, 1689 (2001).

[2] F. Krausz and M. Ivanov, Attosecond physics, Rev. Mod. Phys. 81, 163 (2009).

[3] M. Schultze et al., Delay in photoemission, Science 328, 1658 (2010).

[4] K. Klünder, J. M. Dahlström, M. Gisselbrecht, T. Fordell, M. Swoboda, D. Guénot, P. Johnsson, J. Caillat, J. Mauritsson,

A. Maquet, R. Taïeb, and A. L'Huillier, Probing SinglePhoton Ionization on the Attosecond Time Scale, Phys. Rev. Lett. 106, 143002 (2011).

[5] P. Eckle, M. Smolarski, P. Schlup, J. Biegert, A. Staudte, M. Schöffler, H. G. Muller, R. Dörner, and U. Keller, Attosecond angular streaking, Nat. Phys. 4, 565 (2008).

[6] P. Eckle, A. N. Pfeiffer, C. Cirelli, A. Staudte, R. Dörner, H. G. Muller, M. Büttiker, and U. Keller, Attosecond ionization and tunneling delay time measurements in helium, Science 322, 1525 (2008).

[7] A. N. Pfeiffer, C. Cirelli, M. Smolarski, D. Dimitrovski, M. Abu-samha, L. B. Madsen, and U. Keller, Attoclock reveals natural coordinates of the laser-induced tunneling current flow in atoms, Nat. Phys. 8, 76 (2012).

[8] A. S. Landsman, M. Weger, J. Maurer, R. Boge, A. Ludwig, S. Heuser, C. Cirelli, L. Gallmann, and U. Keller, Ultrafast resolution of tunneling delay time, Optica 1, 343 (2014).

[9] L. Torlina, F. Morales, J. Kaushal, I. Ivanov, A. Kheifets, A. Zielineski, A. Scrinzi, H. G. Muller, S. Sukiasyan, Misha Ivanov, and O. Smirnova, Interpreting attoclock measurements of tunnelling times, Nat. Phys. 11, 503 (2015).

[10] U. S. Sainadh, H. Xu, X. Wang, A. Atia-Tul-Noor, W. C. Wallace, N. Douguet, A. Bray, I. Ivanov, K. Bartschat, A. Kheifets, R. T. Sang, and I. V. Litvinyuk, Attosecond angular streaking and tunnelling time in atomic hydrogen, Nature (London) 568, 75 (2019). 
[11] A. W. Bray, S. Eckart, and A. S. Kheifets, KeldyshRutherford Model for the Attoclock, Phys. Rev. Lett. 121, 123201 (2018).

[12] A. S. Landsman and U. Keller, Attosecond science and the tunnelling time problem, Phys. Rep. 547, 1 (2015).

[13] N. Camus, E. Yakaboylu, L. Fechner, M. Klaiber, M. Laux, Y. Mi, K. Z. Hatsagortsyan, T. Pfeifer, C. H. Keitel, and R. Moshammer, Experimental Evidence for Quantum Tunneling Time, Phys. Rev. Lett. 119, 023201 (2017).

[14] H. Ni, U. Saalmann, and J.-M. Rost, Tunneling Ionization Time Resolved by Backpropagation, Phys. Rev. Lett. 117, 023002 (2016).

[15] N. Eicke and M. Lein, Trajectory-free ionization times in strong-field ionization, Phys. Rev. A 97, 031402(R) (2018).

[16] U.S. Sainadh, Attoclock experiments on atomic and molecular hydrogen, Ph.D. thesis, Griffith University, Brisbane, Australia, 2018.

[17] V. Hanus, S. Kangaparambil, S. Larimian, M. DornerKirchner, X. Xie, M. Schöffler, Gerhard G. Paulus, A. Baltuška, A. Staudte, and M. Kitzler-Zeiler, SubFemtosecond Tracing of Molecular Dynamics During Strong-Field Interaction, arXiv:1907.05347.

[18] J. Ullrich, R. Moshammer, A. Dorn, R. Dörner, L. Ph. H. Schmidt, and H. Schmidt-Böcking, Recoil-ion and electron momentum spectroscopy: Reaction-microscopes, Rep. Prog. Phys. 66, 1463 (2003).

[19] W. Quan, M. H. Yuan, S. G. Yu, S. P. Yu, Y. J. Chen, Y. L. Wang, R. P. Sun, Z. L. Xiao, C. Gong, L. Q. Hua, X. Y. Lai, X. J. Liu, and J. Chen, Laser intensity determination using nonadiabatic tunneling ionization of atoms in close-to-circularly polarized laser fields, Opt. Express 24, 23248 (2016).

[20] Y. L. Wang, S. P. Xu, W. Quan, C. Gong, X. Y. Lai, S. L. Hu, M. Q. Liu, J. Chen, and X. J. Liu, Recoil-ion momentum distribution for nonsequential double ionization of $\mathrm{Xe}$ in intense midinfrared laser fields, Phys. Rev. A 94, 053412 (2016).

[21] W. Quan, X. L. Hao, X. Q. Hu, R. P. Sun, Y. L. Wang, Y. J. Chen, S. G. Yu, S. P. Xu, Z. L. Xiao, X. Y. Lai, X. Y. Li, W. Becker, Y. Wu, J. G. Wang, X. J. Liu, and J. Chen, LaserInduced Inelastic Diffraction from Strong-Field Double Ionization, Phys. Rev. Lett. 119, 243203 (2017).

[22] See Supplemental Material at http://link.aps.org/ supplemental/10.1103/PhysRevLett.123.223204 for details on the experimental setup, the theoretical model, and the data analysis procedure.

[23] V. V. Serov, Calculation of intermediate-energy electronimpact ionization of molecular hydrogen and nitrogen using the paraxial approximation, Phys. Rev. A 84, 062701 (2011).

[24] V. V. Serov and A. S. Kheifets, $p-\mathrm{H}$ symmetry breaking in dissociative photoionization of $\mathrm{H}_{2}$ due to the molecular ion interacting with the ejected electron, Phys. Rev. A 89, 031402(R) (2014).

[25] T. Zimmermann, S. Mishra, B. R. Doran, D. F. Gordon, and A. S. Landsman, Tunneling Time and Weak Measurement in Strong Field Ionization, Phys. Rev. Lett. 116, 233603 (2016).

[26] Y. Shao, P. L. He, M. M. Liu, X. F. Sun, M. Li, Y. K. Deng, C. Y. Wu, F. He, Q. H. Gong, and Y. Q. Liu, Fully differential study on dissociative ionization dynamics of deuteron molecules in strong elliptically laser fields, Phys. Rev. A 95, 031404(R) (2017).

[27] M. Kunitski, N. Eicke, P. Huber, J. Köhler, S. Zeller, J. Voigtsberger, N. Schlott, K. Henrichs, H. Sann, F. Trinter, L. Ph. H. Schmidt, A. Kalini, M. S. Schöffler, T. Jahnke, M. Lein, and R. Dörner, Double-slit photoelectron interference in strong-field ionization of the neon dimer, Nat. Commun. 10, 1 (2019). 